\title{
Amelioration of the Development of Osteoarthritis by Daily Intake of $\beta$-Cryptoxanthin
}

\author{
Gyujin Park, ${ }^{a, \#}$ Tetsuhiro Horie,,${ }^{a, \#}$ Kazuya Fukasawa, ${ }^{a}$ Kakeru Ozaki, ${ }^{a}$ Yuki Onishi, ${ }^{a}$ \\ Takashi Kanayama, ${ }^{a}$ Takashi Iezaki, ${ }^{a, b}$ Katsuyuki Kaneda ${ }^{a}{ }^{\text {Minoru Sugiura }},{ }^{c}$ and Eiichi Hinoi*, \\ ${ }^{a}$ Laboratory of Molecular Pharmacology, Division of Pharmaceutical Sciences, Kanazawa University Graduate \\ School; Kakuma-machi, Kanazawa, Ishikawa 920-1192, Japan: ${ }^{b}$ Venture Business Laboratory, Organization of \\ Frontier Science and Innovation, Kanazawa University; Kakuma-machi, Kanazawa, Ishikawa 920-1192, Japan: and \\ ${ }^{c}$ Citrus Research Division, Institute of Fruit Tree and Tea Science, National Agriculture and Food Research \\ Organization; 485-6 Okitsunaka-cho, Shimizu, Shizuoka 424-0292, Japan.
}

Received February 20, 2017; accepted April 4, 2017

\begin{abstract}
$\beta$-Cryptoxanthin, which is primarily obtained from citrus fruits such as Satsuma mandarins, is a major carotenoid routinely found in human serum. Recently, we demonstrated that daily oral intake of $\beta$-cryptoxanthin prevented ovariectomy-induced bone loss and ameliorated neuropathic pain in mice. Although $\beta$-cryptoxanthin exerts preventive effects on various lifestyle-related diseases, there have been no studies on the effect of $\beta$-cryptoxanthin on the development of osteoarthritis, the most common degenerative joint disease, which frequently leads to loss of ability and stiffness in the elderly. Here we showed that daily oral administration of $\beta$-cryptoxanthin significantly prevented the development of osteoarthritis developed by surgically inducing knee joint instability in mice in vivo. Furthermore, in vitro experiments revealed that $\beta$-cryptoxanthin markedly inhibited the expression of inflammatory cytokines and enzymes critical for the degradation of the extracellular matrix in primary chondrocytes. Our results suggest that oral supplementation of $\beta$-cryptoxanthin would be beneficial for the maintenance of joint health and as prophylaxis against osteoarthritis.
\end{abstract}

Key words osteoarthritis; $\beta$-cryptoxanthin; chondrocyte; inflammatory cytokine

\begin{abstract}
$\beta$-Cryptoxanthin, which has eight isoprene units with $\beta$ rings, is primarily obtained from citrus fruits such as Satsuma mandarin (Citrus unshiu MARC.), and is a major carotenoid routinely found in the human serum. ${ }^{1,2)}$ In humans and animals, the serum level of $\beta$-cryptoxanthin reflects the amount of intake of $\beta$-cryptoxanthin-rich foods. ${ }^{3)}$ We recently demonstrated that $\beta$-cryptoxanthin prevented ovariectomy-induced osteoclast activation and bone loss and ameliorated the development of tactile allodynia following spinal nerve injury in mice. ${ }^{4,5}$ Although accumulating evidence indicates a preventive effect of $\beta$-cryptoxanthin on various lifestyle-related diseases, thus far, few studies are available on the effect of $\beta$-cryptoxanthin on the development of osteoarthritis (OA), which is the most common degenerative joint disease, affecting approximately 250 million people worldwide. ${ }^{\text {) }}$
\end{abstract}

$\mathrm{OA}$, which is characterized by progressive articular cartilage destruction, is caused by various factors, including joint injury, obesity, and aging, and frequently leads to a loss of ability and stiffness in the elderly. ${ }^{7}$ Thus far, no effective medical therapies or preventive supplementations have been developed that prevent OA cartilage destruction, and new therapeutic and preventive approaches to reduce joint pain and slow the progression of the condition are desirable. Supplementation of the cartilage constituents glucosamine and chondroitin sulfates has been used to relieve pain or slow the degradation of cartilage in joints ${ }^{8,9}$; however, it has recently been reported that glucosamine and chondroitin sulfates, even in combination, do not have a clinically relevant effect on perceived joint pain or on joint space narrowing. ${ }^{10)}$ Furthermore,

\footnotetext{
\# These authors contributed equally to this work.

long-term treatment of patients with OA with analgesics and non-steroidal anti-inflammatory drugs can lead to serious gastrointestinal and cardiovascular adverse events.

In this study, we investigated pharmacological properties of $\beta$-cryptoxanthin administered to mice with surgically induced cartilage destruction as a model of OA in vivo. Our observations suggest that $\beta$-cryptoxanthin supplementation could suppress the development of OA generated by surgically inducing knee joint instability in mice.

\section{MATERIALS AND METHODS}

Materials Nonesterified $\beta$-cryptoxanthin used in this study was prepared and processed as described previously, and its purity was $96 \%$ according to HPLC analysis. ${ }^{11)} \mathrm{Re}-$ combinant mouse interleukin-1 $\beta$ (IL-1 $\beta$ ) was purchased from Cell Signaling Technology (U.S.A.). THUNDERBIRD SYBR qPCR Mix was supplied by TOYOBO (Japan). All other chemicals used were of the highest purity available commercially.

Mice and OA Model The protocol used here meets the guideline of the Japanese Society for Pharmacology and was approved by the Committee for Ethical Use of Experimental Animals at Kanazawa University. OA was surgically induced in 8 week-old male C57BL/6J mice by destabilizing the knee joint as previously described. ${ }^{12)}$ The right knee joint was destabilized by transection of the medial collateral ligament and removal of the cranial half of the medial meniscus under general anesthesia. A sham operation was performed on the left knee joint. For histological analysis, mouse knee joints were fixed with $10 \%$ formalin neutral buffer, and subsequent decal- 
(A) Waker intake

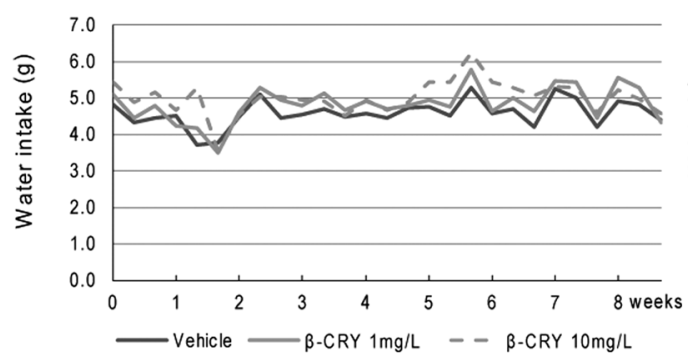

(B) Body weight

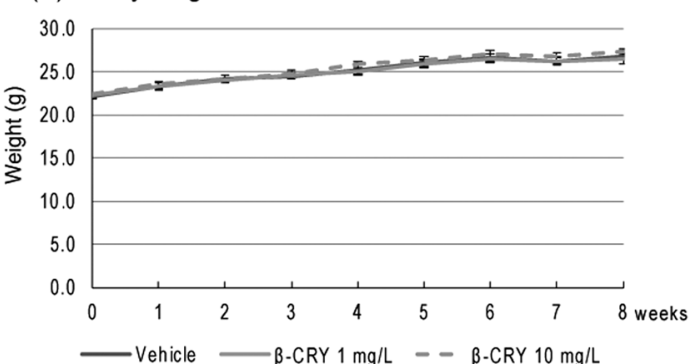

(C)

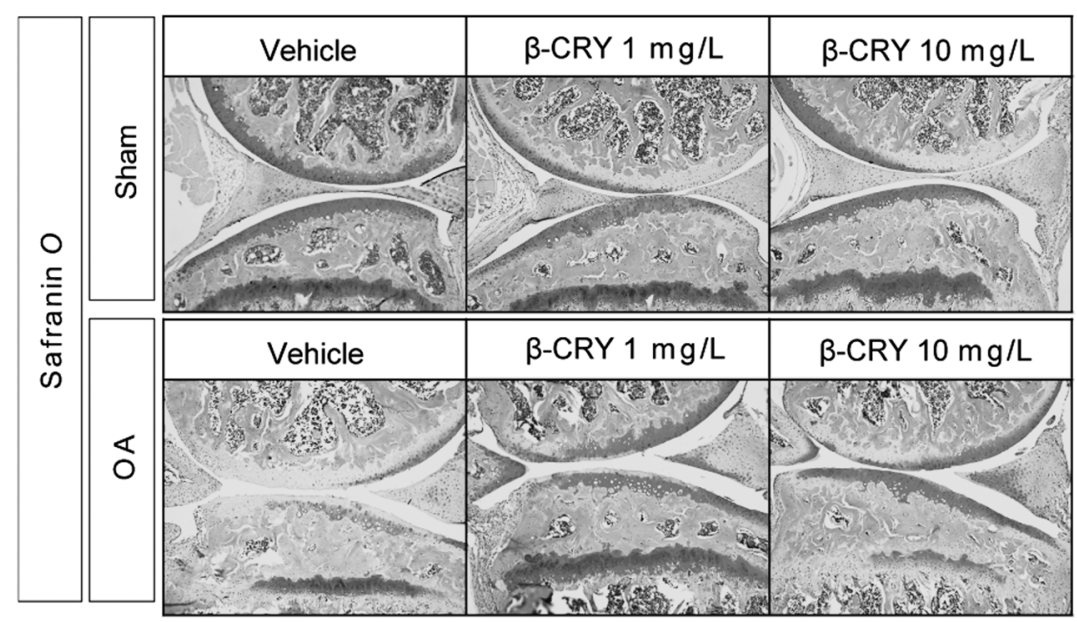

(D)

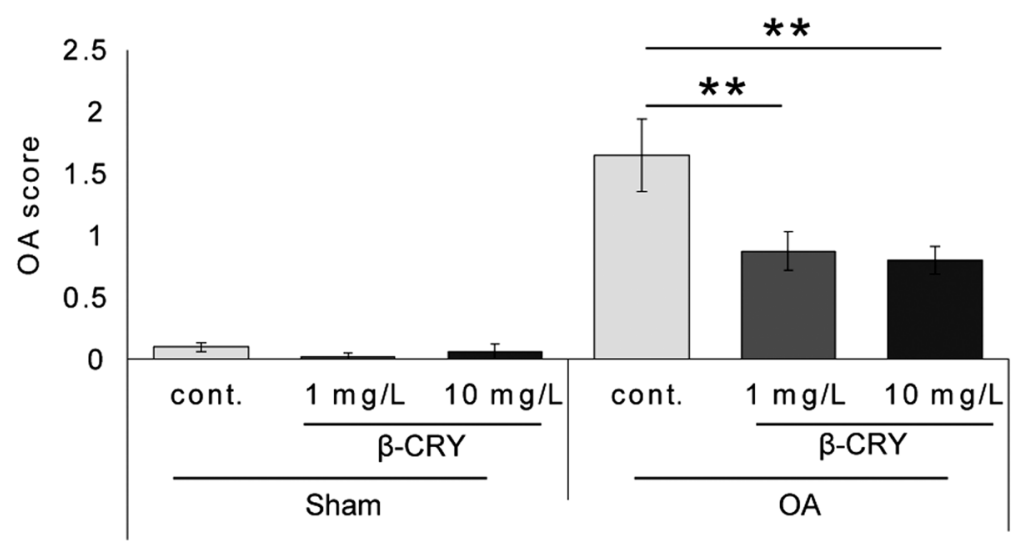

Fig. 1. Oral Supplementation of $\beta$-Cryptoxanthin $(\beta$-CRY) Ameliorates the Development of OA

Eight-week-old male $\mathrm{C} 57 \mathrm{BL} / 6 \mathrm{~J}$ mice were subjected to $\mathrm{OA}$, followed by daily oral supplementation of $\beta$-CRY at $1 \mathrm{mg} / \mathrm{L}$ and $10 \mathrm{mg} / \mathrm{L}$ for 8 consecutive weeks (vehicle, $n=15 ; \beta$-CRY $1 \mathrm{mg} / \mathrm{L}, n=15 ; \beta$-CRY $10 \mathrm{mg} / \mathrm{L}, n=16$ ). (A) Water intake and (B) body weight. Typical pictures of Safranin O staining are shown in the panel (C), while quantitative OA score are shown in the panel (D). ${ }^{* *} p<0.01$, significantly different from control value obtained in joint of surgical-induced OA of vehicle-treated mice.

Table 1. List of Primers Used for RT-qPCR

\begin{tabular}{lll}
\hline \hline Genes & \multicolumn{1}{c}{ Upstream $\left(5^{\prime}-3^{\prime}\right)$} & \multicolumn{1}{c}{ Downstream $\left(5^{\prime}-3^{\prime}\right)$} \\
\hline Adamts 5 & CCCAGGATAAAACCAGGCAG & CGGCCAAGGGTTGTAAATGG \\
$I l 1 b$ & GAAATGCCACCTTTTGACAGTG & CTGGATGCTCTCATCAGGACA \\
$I l 6$ & ATTCCAGAAACCGCTATGAA & TGCCATTGCACAACTCTTTT \\
Tmp 13 & AGGCCTTCAGAAAAGCCTTC & TCCTTGGAGTGATCCAGACC \\
$36 b 4$ & GTAGCCCACGTCGTAGCAAAC & CTGGCACCACTAGTTGGTTGTC \\
\hline
\end{tabular}

cification with $20 \%$ ethylenediaminetetraacetic acid (EDTA) ( $\mathrm{pH}$ 7.4) for 1 week, followed by embedding in paraffin. Knee joints were then dissected for sections with a thickness of
$5 \mu \mathrm{m}$ using a microtome (Leica RM2125 RTS), and subsequently sections were stained with Safranin O and Fast Green. OA severity was quantified by the Osteoarthritis Research So- 

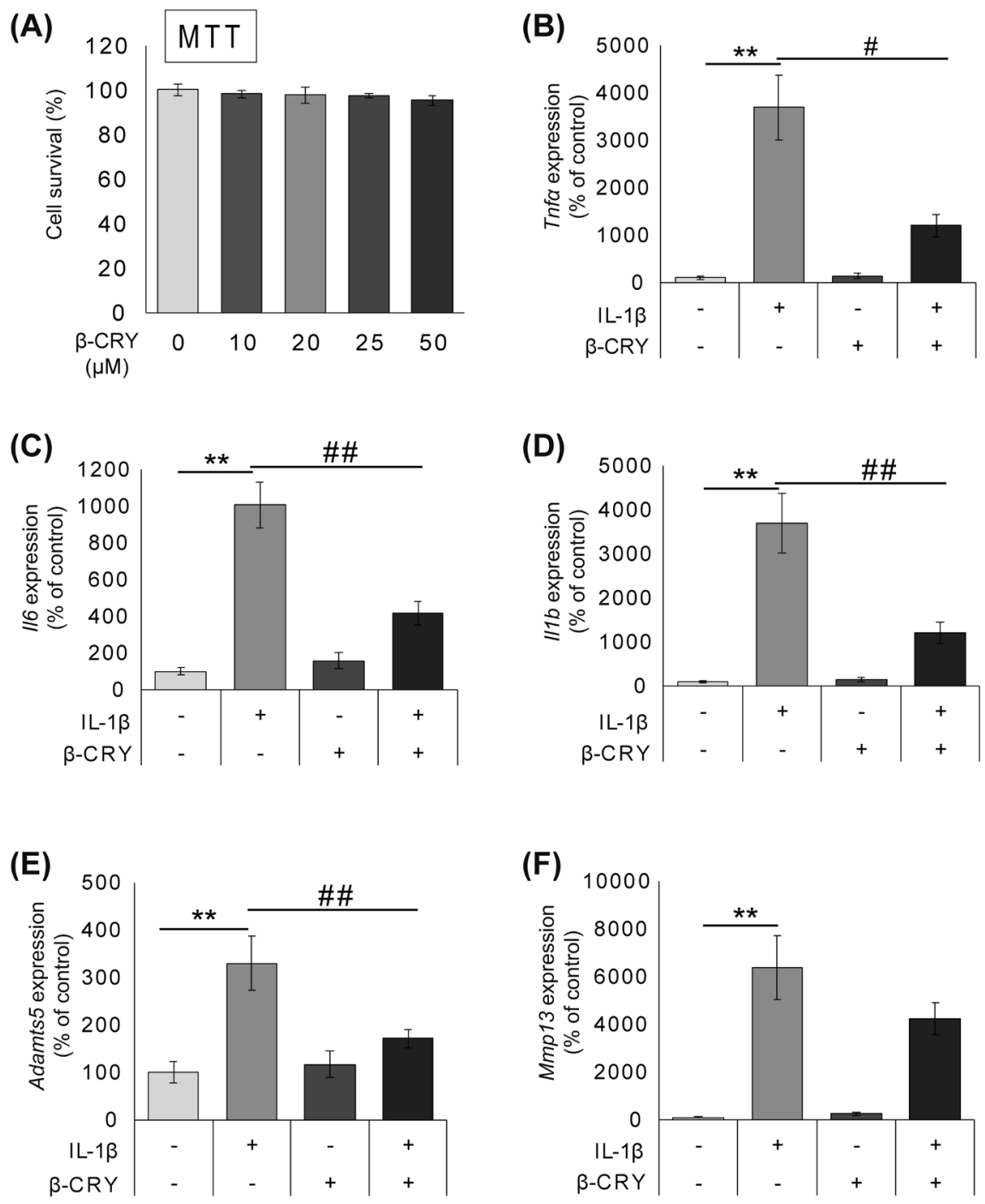

Fig. 2. $\beta$-CRY Inhibits the Expression of Inflammatory Cytokines in Chondrocytes without Affecting Cell Survival

(A) Primary chondrocytes were cultured with $\beta$-CRY at a concentration range from 10 to $50 \mu \mathrm{m}$, followed by determination of MTT reduction ( $n=4$ ). Primary chondrocytes were cultured with IL-1 $\beta$ at $10 \mathrm{ng} / \mathrm{mL}$ in either the presence or absence of $\beta$-CRY at $50 \mu \mathrm{m}$, followed by determination of mRNA expression of (B) Tnfa, (C) Il6, (D) Illb, (E) Adamts5, and (F) Mmpl3 (n=10-17). ${ }^{* *} p<0.01$, significantly different from each control value obtained in the absence of both IL-1 $\beta$ and $\beta$-CRY. ${ }^{\#} p<0.05$, $\# p<0.01$, significantly different from the value obtained for cells treated with IL-1 $\beta$ alone.

ciety International (OARSI) histopathology grading system. ${ }^{13)}$ Articular surfaces (femoral condyles and tibial plateaus) were graded. OA model mice received daily oral supplementation of $\beta$-cryptoxanthin freshly dissolved in drinking water containing $0.5 \%$ polysorbate 80 at concentrations of 1 or $10 \mathrm{mg} / \mathrm{L}$ for 8 weeks. Vehicle solution (drinking water containing $0.5 \%$ polysorbate 80) was used for control.

Culture of Primary Chondrocytes Mouse primary chondrocytes were prepared from thoracic cages of pups at postnatal 3-5d old by the sequential enzymatic digestion method as described previously. ${ }^{14)}$ In brief, thoracic cages were dissected and subsequently incubated with $0.3 \%$ collagenase for $90 \mathrm{~min}$ to remove soft tissues. The thoracic cages were further digested with $0.3 \%$ collagenase for $6 \mathrm{~h}$. A differentiation inducer mixture containing $50 \mu \mathrm{g} / \mathrm{mL}$ ascorbic acid, $1 \mathrm{~mm}$ pyruvate, and $1 \mathrm{~mm}$ cysteine was used for the culture of primary chondrocytes.

The 3-(4,5-dimethylthiazol-2-yl)-2,5-diphenyl-2H-tetrazolium bromide (MTT) assay were performed, as previously described. ${ }^{15)}$
RT-qPCR Total RNA was extracted from cells, followed by synthesis of cDNA with reverse transcriptase and oligo-dT primer. ${ }^{16)}$ The cDNA samples were then used as templates for real-time PCR analysis, which was performed on an MX3005P instrument (Agilent Technologies), by using specific primers for each gene (Table 1). Expression levels of the genes examined were normalized by using the $36 \mathrm{~b} 4$ expression levels as an internal control for each sample.

Data Analysis Results are all expressed as the mean \pm standard error (S.E.) and the statistical significance was determined by the one-way ANOVA with Bonferroni/Dunnett post hoc test.

\section{RESULTS}

Oral Supplementation of $\boldsymbol{\beta}$-Cryptoxanthin Ameliorated the Development of $\mathbf{O A}$ To examine whether daily oral intake of $\beta$-cryptoxanthin could modulate the development of OA, we administered $\beta$-cryptoxanthin orally in drinking water to OA model mice for 8 weeks at concentrations of 1 
or $10 \mathrm{mg} / \mathrm{L}$. No significant change was observed in the daily intake of drinking water by the mice, irrespective of the concentrations of $\beta$-cryptoxanthin used (Fig. 1A). In addition, $\beta$-cryptoxanthin administration did not affect the body weight of the mice (Fig. 1B) or their spontaneous behavior (data not shown). Under these experimental conditions, no marked morphological differences in sham-operated joints were detected between the vehicle-treated and $\beta$-cryptoxanthin-treated mice (Fig. 1C, upper three panels). In the vehicle-treated mice, marked cartilage degradation was observed by Safranin $\mathrm{O}$ staining in joints with surgical-induced OA compared with that in sham-operated joints (Fig. 1C, left two panels). Conversely, a marked amelioration of cartilage degradation was observed in the joints with surgical-induced OA when $\beta$-cryptoxanthin was administered at both 1 and $10 \mathrm{mg} / \mathrm{L}$ (Fig. $1 \mathrm{C}$ lower three panels). We also analyzed data using the histologic scoring system recommended by OARSI; this showed that mice treated with $\beta$-cryptoxanthin at both 1 and $10 \mathrm{mg} / \mathrm{L}$ had significantly lower scores for OA damage than vehicletreated mice (Fig. 1D). These results indicate that daily oral $\beta$-cryptoxanthin treatment suppressed the development of OA.

$\boldsymbol{\beta}$-Cryptoxanthin Inhibited the Expression of Inflammatory Cytokines in Chondrocytes Pro-inflammatory signals lead to the activation of several pathways involving the production of pro-inflammatory cytokines themselves and degradation of the extracellular matrix (ECM) by matrix metalloproteinases (MMPs) and a disintegrin and metalloproteinase with thrombospondin motifs (ADAMTS) in chondrocytes. ${ }^{17)}$ To clarify the cellular mechanisms underlying the preventive effect of $\beta$-cryptoxanthin on $\mathrm{OA}$ development in vivo, we investigated its effect on gene expression profiles in chondrocytes in vitro. Treatment with $\beta$-cryptoxanthin at the concentrations tested (even at $50 \mu \mathrm{M}$ ) did not affect MTT reduction, as an index of cellular survival in chondrocytes (Fig. 2A). We, therefore, stimulated primary chondrocytes with IL- $1 \beta$ at $10 \mathrm{ng} / \mathrm{mL}$ in the presence or absence of $\beta$-cryptoxanthin at $50 \mu \mathrm{M}$, and followed this with the determination of gene expression. IL-1 $\beta$ markedly elevated the expression of inflammatory cytokines, Tnfa, Il6, and $I l 1 b$ in chondrocytes, whereas these inductions were significantly impaired in chondrocytes treated with $\beta$-cryptoxanthin (Figs. 2B-D). Furthermore, $\beta$-cryptoxanthin significantly inhibited the expression of IL-1 $\beta$-induced Adamts 5 expression in chondrocytes (Fig. 2E), whereas we observed a trend toward inhibition of the expression of Mmpl3 by $\beta$-cryptoxanthin (Fig. $2 \mathrm{~F}$ ), although this effect was not statistically significant.

\section{DISCUSSION}

Knee OA is one of the major locomotive disorders, often requiring special assistance or nursing care in elderly individuals. Several independent lines of evidence have demonstrated that $\beta$-cryptoxanthin has a preventive effect on various lifestyle-related diseases, including osteoporosis and diet-induced nonalcoholic steatohepatitis. ${ }^{11,18)}$ Previous studies have indicated that $\beta$-cryptoxanthin may be useful in blocking the progression of arthritis including rheumatoid arthritis (RA) and OA. However, they showed that $\beta$-cryptoxanthin can exert anti-arthritic effect in antigen-induced arthritic rats in vivo as a model of RA but not of OA. ${ }^{19)} \mathrm{OA}$ and RA are different types of arthritis, with the main difference between them being the underlying cause of the joint symptoms: OA is caused by mechanical wear and tear on joints, whereas RA is an autoimmune disease. ${ }^{20,21)}$ An important result of the present study is that daily oral supplementation of the xanthophyll carotenoid $\beta$-cryptoxanthin significantly prevented the development of surgically-induced OA but not of RA in vivo. Furthermore, it should be emphasized that we have revealed, for the first time, that $\beta$-cryptoxanthin markedly inhibited the IL- $1 \beta$-induced expression of inflammatory cytokines (Tnfa, Il6, and Illb) in primary chondrocytes. It is well known that inflammatory cytokines mediate OA development by upregulating the expression of cartilage-degrading enzymes, such as MMP13 and ADAMTS5, which are primary enzymes responsible for degradation of type II collagen and aggrecan, respectively, in articular cartilage. ${ }^{22)}$ Although further studies are needed to reveal the underlying mechanisms of the protective effects of $\beta$-cryptoxanthin against surgically induced cartilage degeneration, to the best of our knowledge, the present study is the first to demonstrate the modulation of $\mathrm{OA}$ by $\beta$-cryptoxanthin.

In this study, we showed that the development of OA was ameliorated by daily administration of $\beta$-cryptoxanthin at $1 \mathrm{mg} / \mathrm{L}$ for 8 consecutive weeks. As the mice were administered $5 \mathrm{~mL} / \mathrm{d}$ of drinking water, under our experimental protocols they should be administered $9.0 \mathrm{nmol}$ of $\beta$-cryptoxanthin daily. It has been established that $\beta$-cryptoxanthin forms retinol (vitamin A), which plays a pivotal role in a variety of biological functions including anti-oxidant and anti-inflammation. ${ }^{2)}$ Although it should be considered that $\beta$-cryptoxanthin may exert on OA through its metabolites, long-term intake of $\beta$-cryptoxanthin-rich foods gradually increases blood $\beta$ cryptoxanthin levels in both humans and animals. ${ }^{3)}$ It is, therefore, conceivable that the daily consumption of $\beta$ cryptoxanthin-rich foods could achieve a pharmacologically relevant dose of $\beta$-cryptoxanthin similar to that used in this study. Accordingly, we propose that appropriate consumption of $\beta$-cryptoxanthin-rich foods, such as Satsuma mandarins (Citrus unshiu MARC.), would be beneficial for the maintenance of joint health and as prophylaxis against OA.

Acknowledgments This work was supported in part by Grants-in-Aid for Scientific Research to E.H. from the Ministry of Education, Culture, Sports, Science and Technology of Japan.

Conflict of Interest The authors declare no conflict of interest.

\section{REFERENCES}

1) Sugiura M. beta-Cryptoxanthin and the risk for lifestyle-related disease: findings from recent nutritional epidemiologic studies. Yakugaku Zasshi, 135, 67-76 (2015).

2) Burri BJ. Beta-cryptoxanthin as a source of vitamin A. J. Sci. Food Agric., 95, 1786-1794 (2015).

3) Sugiura M. beta-Cryptoxanthin and the risk for lifestyle-related disease: findings from recent nutritional epidemiologic studies. Yakugaku Zasshi, 135, 67-76 (2015).

4) Ozaki K, Okamoto M, Fukasawa K, Iezaki T, Onishi Y, Yoneda Y, Sugiura M, Hinoi E. Daily intake of beta-cryptoxanthin prevents bone loss by preferential disturbance of osteoclastic activation in ovariectomized mice. J. Pharmacol. Sci., 129, 72-77 (2015).

5) Park G, Horie T, Iezaki T, Okamoto M, Fukasawa K, Kanayama T, 
Ozaki K, Onishi Y, Sugiura M, Hinoi E. Daily oral intake of betacryptoxanthin ameliorates neuropathic pain. Biosci. Biotechnol. Biochem., 81, 1014-1017 (2017).

6) Shen J, Chen D. Recent progress in osteoarthritis research. J. Am. Acad. Orthop. Surg., 22, 467-468 (2014).

7) Tsezou A. Osteoarthritis year in review 2014: genetics and genomics. Osteoarthritis and cartilage/OARS, Osteoarthritis Research Society, 22, 2017-2024 (2014).

8) Clegg DO, Reda DJ, Harris CL, Klein MA, O'Dell JR, Hooper MM, Bradley JD, Bingham CO 3rd, Weisman MH, Jackson CG, Lane NE, Cush JJ, Moreland LW, Schumacher HR Jr, Oddis CV, Wolfe F, Molitor JA, Yocum DE, Schnitzer TJ, Furst DE, Sawitzke AD, Shi H, Brandt KD, Moskowitz RW, Williams HJ. Glucosamine, chondroitin sulfate, and the two in combination for painful knee osteoarthritis. N. Engl. J. Med., 354, 795-808 (2006).

9) Bruyere O, Reginster JY. Glucosamine and chondroitin sulfate as therapeutic agents for knee and hip osteoarthritis. Drugs Aging, 24, 573-580 (2007).

10) Vasiliadis HS, Tsikopoulos K. Glucosamine and chondroitin for the treatment of osteoarthritis. World J. Orthop., 8, 1-11 (2017).

11) Kobori M, Ni Y, Takahashi Y, Watanabe N, Sugiura M, Ogawa K, Nagashimada M, Kaneko S, Naito S, Ota T. beta-Cryptoxanthin alleviates diet-induced nonalcoholic steatohepatitis by suppressing inflammatory gene expression in mice. PLOS ONE, 9, e98294 (2014).

12) Iezaki $T$, Ozaki $K$, Fukasawa $K$, Inoue $M$, Kitajima $S$, Muneta $T$, Takeda S, Fujita H, Onishi Y, Horie T, Yoneda Y, Takarada T, Hinoi E. ATF3 deficiency in chondrocytes alleviates osteoarthritis development. J. Pathol., 239, 426-437 (2016).

13) Glasson SS, Chambers MG, Van Den Berg WB, Little CB. The OARSI histopathology initiative-recommendations for histological assessments of osteoarthritis in the mouse. Osteoarthritis and cartilage/OARS, Osteoarthritis Research Society, 18 (Suppl. 3), S17-S23 (2010).

14) Wang L, Hinoi E, Takemori A, Takarada T, Yoneda Y. Abolition of chondral mineralization by group III metabotropic glutamate receptors expressed in rodent cartilage. Br. J. Pharmacol., 146, 732-743 (2005).

15) Yamamoto T, Hinoi E, Fujita H, Iezaki T, Takahata Y, Takamori $\mathrm{M}$, Yoneda $\mathrm{Y}$. The natural polyamines spermidine and spermine prevent bone loss through preferential disruption of osteoclastic activation in ovariectomized mice. Br. J. Pharmacol., 166, 1084-1096 (2012).

16) Fujimori S, Hinoi E, Yoneda Y. Functional GABA(B) receptors expressed in cultured calvarial osteoblasts. Biochem. Biophys. Res. Commun., 293, 1445-1452 (2002).

17) Song RH, Tortorella MD, Malfait AM, Alston JT, Yang Z, Arner EC, Griggs DW. Aggrecan degradation in human articular cartilage explants is mediated by both ADAMTS-4 and ADAMTS-5. Arthritis Rheum., 56, 575-585 (2007).

18) Sugiura M, Nakamura M, Ogawa K, Ikoma Y, Yano M. High serum carotenoids associated with lower risk for bone loss and osteoporosis in post-menopausal Japanese female subjects: prospective cohort study. PLOS ONE, 7, e52643 (2012).

19) Imada $K$, Tsuchida A, Ogawa K, Sofat N, Nagase H, Ito A, Sato T. Anti-arthritic actions of beta-cryptoxanthin against the degradation of articular cartilage in vivo and in vitro. Biochem. Biophys. Res. Commun., 476, 352-358 (2016)

20) Tsuruha J, Masuko-Hongo K, Kato T, Sakata M, Nakamura H, Nishioka K. Implication of cartilage intermediate layer protein in cartilage destruction in subsets of patients with osteoarthritis and rheumatoid arthritis. Arthritis Rheum., 44, 838-845 (2001).

21) Pap T, Korb-Pap A. Cartilage damage in osteoarthritis and rheumatoid arthritis-two unequal siblings. Nat. Rev. Rheumatology, 11, 606-615 (2015).

22) Wang M, Sampson ER, Jin H, Li J, Ke QH, Im HJ, Chen D. MMP13 is a critical target gene during the progression of osteoarthritis. Arthritis Res. Ther., 15, R5 (2013). 